\title{
Histopathological Diagnosis of Cervical Biopsies: Reduction of Sampling Errors with the Evaluation of a Third Histologic Level
}

This article was published in the following Dove Press journal: Cancer Management and Research

\begin{abstract}
Edgar Villegas-Hinojosa (iD) Yolanda Terán-Figueroa (iD) Veronica Gallegos-García (D) ' Dario Gaytán-Hernández (iD) Sandra O Gutiérrez-Enríquez (iD) Anahid E Campuzano-Barajas ${ }^{2}$ Luz E Alcántara-Quintana (iD) ${ }^{3}$

'Facultad de Enfermería y Nutrición, Universidad Autónoma de San Luis, Potosí 78240, SLP, México; ${ }^{2}$ Hospital General de Soledad, Secretaría de Salud, Soledad de Graciano Sánchez 78435, SLP, México; ${ }^{3}$ Cátedra CONACYT, adscrito a Facultad de Enfermería y Nutrición, Universidad Autónoma de San Luis, Potosí 78240, SLP, México
\end{abstract}

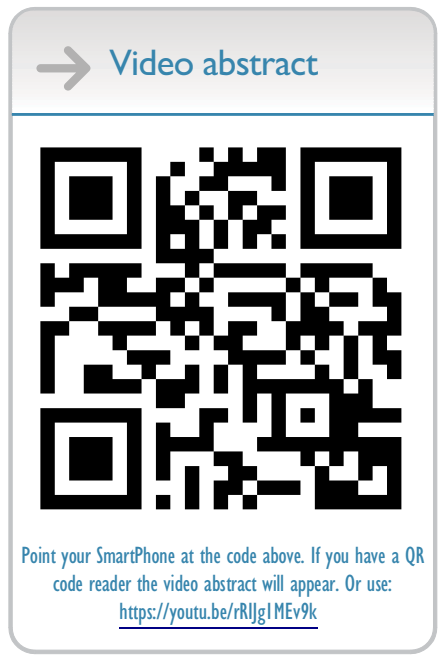

Correspondence: Luz E AlcántaraQuintana

Facultad de Enfermeria y Nutrición, UASLP,Av. Niño Artillero 130 Zona Universitaria,SLP 78240,SLP

Tel +52 4448262300 ext 5032

Email luz.alcantara@uaslp.mx

\begin{abstract}
Aim: Cervical cancer (CC) is considered as a major public health problem; this disease affects mainly vulnerable women in poverty, causing a negative effect on a country's workforce.

Objective: To determine the histopathological diagnosis variation after examining three more levels of cervical biopsy paraffin blocks from patients with HPVI, CIN and CC.

Materials and Methods: A quantitative, retrospective correlational study was performed in a hospital with a second level of health care. We worked with 152 paraffin blocks of CIN (CIN, I, II and III) and cervical cancer samples. Currently, CIN I is considered as a set of low-grade injuries (low-grade squamous intraepithelial lesions, LSIL), while CIN II, CIN III and cancer in situ are considered as high-grade lesions (high-grade squamous intraepithelial lesions, HSIL). A slab was prepared with the 50micron block, which was subsequently cut into 5 microns; later, the same thing was done at two more levels to reevaluate the histopathological diagnosis and correlate it with the initial diagnosis issued by the institution. Results: During the examination of the additional block levels, a difference was observed from the initial diagnosis: of 32 cases of HPV diagnosis, there were 17 changes to CIN I; of 31 cases of CIN I, there were 4 changes to CIN II; of 30 cases of CIN II, there were 8 changes to CIN III and 1 change to invasive cancer; of 29 cases of CIN III, there were 9 changes to cancer in situ; and finally, of 14 cases of cancer in situ, there was 1 change to invasive cancer. After the statistical analysis, a value of $\mathrm{p}<0.05$ was obtained, which indicated that the differences were statistically significant.
\end{abstract}

Conclusion: By modifying the histopathological study technique, guidelines can be given to generate a more accurate diagnosis with a more solid base, and thus, a more appropriate and timely treatment can be offered to avoid the development of cervical cancer.

Keywords: histopathological diagnosis, levels, cervical intraepithelial neoplasia, CIN, cervical cancer, low-grade squamous intraepithelial lesion, LSIL, high-grade squamous intraepithelial lesion, HSIL

\section{Introduction}

Cervical cancer (CC) is considered a major public health problem; this disease affects mainly vulnerable women in poverty, causing a negative effect on a country's workforce. Families and communities are also affected, since they can lose prematurely their family heads, mothers, wives and grandmothers, who have important and irreplaceable roles in society. ${ }^{1}$

Effective methods for the early detection of precancerous lesions such as Papanicolaou (PAP), colposcopy, histopathological study, hybrid capture and PCR have helped to reduce the recurrence of invasive $\mathrm{CC}$. The assessment of patients by 
means of these methods allows the differentiation between sick and healthy women. ${ }^{2}$ The use of combined methods for the detection of CC improves the degree of confidence in such diagnoses.

Histopathology is the reference standard for the diagnosis of cervical intraepithelial neoplasms (CIN) and CC; and this analysis will determine how women will be treated, as well as the follow-up they will receive. ${ }^{3}$ Currently CIN I is considered as a set of low-grade injuries (lowgrade squamous intraepithelial lesions, LSIL), while CIN II, CIN III and cancer in situ are considered high-grade lesions (high-grade squamous intraepithelial lesions, HSIL). Nevertheless, certain methodological improvements have been suggested; previous studies have reported that the sensitivity of a histopathological study may increase by making a greater number of cuts at different levels in the paraffin block of the cervical biopsy. ${ }^{4-6}$

Based on the above, the objective of this work was to determine the variation in a histopathological diagnosis ${ }^{7,8}$ when examining three more levels in the paraffin blocks of biopsies from patients infected with Human Papillomavirus (HPVI), at different CIN and CC stages. The purpose was to obtain a more accurate diagnosis at a histopathological level by examining more levels in the biopsy paraffin blocks and comparing them with the initial diagnoses. ${ }^{9-12}$

\section{Materials and Methods}

This was a quantitative, correlational and retrospective study. A total of 152 paraffin blocks were analyzed from cervical biopsies of patients diagnosed with HPVI $(n=32)$, CIN I ( $n=31)$, CINII ( $n=30)$, CIN III $(n=29)$, in situ CC $(n=14)$ and invasive $\mathrm{CC}(\mathrm{n}=16)$. These samples were obtained from the pathology service at a hospital with a second level of health care (Hospital General de Soledad) and have biopsy dates prior to 2014. The criterion for including the samples in the study was that the paraffin blocks belonged to patients with some degree of CIN, HPVI, and CC; and the criterion for excluding them was that the samples were obtained after 2014.

The initial diagnosis was established by the pathologist of the institution from a single cut in the first level of the paraffin block (Figure 1). The study was carried out with prior authorization from the Hospital Ethics Committee, registration: CEI-HGS-001-20, and ARCO rights, article 16, second paragraph of the Mexican Constitution, which establishes that everyone has the right to safeguard their personal information. All the medical research was carried out at the Hospital General de Soledad, in San Luis Potosí,

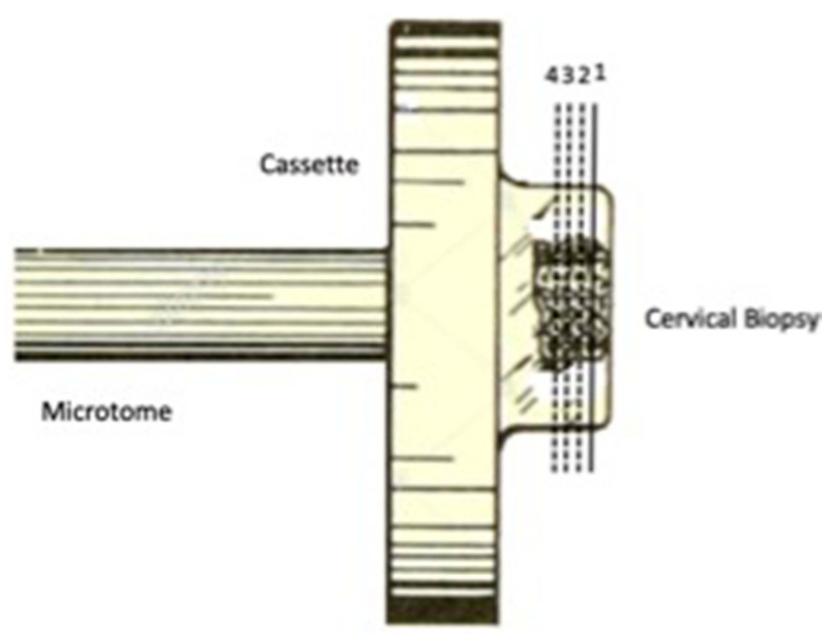

Figure I Block of paraffin and cuts at different levels. The lines and numbers mark the cut levels from the first to the fourth. The cuts were made before reaching each line. The continuous line shows the first level; this is where the first cut was made by the original institution pathologist and represents the sample with which the initial diagnosis was established. The dotted lines show the second to the third levels. The numbers mark the sites where slabs and cuts were made to reassess the initial diagnosis.

Mexico; and strictly followed the principles established in the Declaration of Helsinki.

In Mexico, paraffin blocks from samples that have been in storage for more than five years are discarded. So, no additional permission was required from the hospital ethics committee.

Cuts at different levels in the paraffin blocks of the cervical biopsies were made with a microtome (Leica Biosystems, RM-2125-RTS, Germany) at an angle of 5 to $7^{\circ}$ from the base. From the first level of the block, a roughing of 50 microns was made, and a cut of 3 to 5 microns was made in the second level. Subsequently, the procedure was repeated to generate another cut on the third and fourth levels of the block (Figure 1). The sections were stretched on a surface in a water bath (Premiere, tissue floating bath, $\mathrm{XH}-1001$, USA) at $45^{\circ} \mathrm{C}$ and then mounted on a slide, dried at $65^{\circ} \mathrm{C}$ in a drying chamber (Binder, ED-115, Germany), stained with hematoxylin-eosin (Merck KGaA, 1050175, Germany) (Hycel, 686, Mexico) and mounted in resin (Sigma Aldrich-506 epoxy resin).

\section{Histopathological Diagnosis}

The Bethesda system was used to analyze the slides of the second, third and fourth levels of the cervical biopsy paraffin block. The histopathological diagnosis was made by two certified pathologists that performed a double-blind analysis under an optical microscope (Leica Biosystems, 
DM-750, $\mathrm{USA}^{2}$ ) at $10 \mathrm{x}$ and $40 \mathrm{x}$ magnifications. The first pathologist performed the observation of all slides, and the second pathologist analyzed only 1 out of 10 slides chosen at random as the gold standard.

\section{Statistical Analysis}

The data processing was performed with the SPSS v. Software. 22.0. and the graphs were generated in Prism v 8. An analysis was performed using descriptive inferential statistics; and the statistical chi-square test was used to evaluate the difference in the proportions between the initial diagnoses made by the institute's pathologist, and the diagnoses obtained when examining different levels in the paraffin blocks of this study. The Kappa coefficient and a 95\% confidence interval were obtained for the correlation between the diagnoses of the first pathologist and the diagnoses of the second pathologist.

\section{Results}

We found out that $36.8 \%$ (56 paraffin blocks, Figure 2) of the diagnoses based on the evaluation of different levels of the biopsy paraffin blocks, differed from the initial diagnoses. When observing and analyzing the slides, it was realized that $12.5 \%(\mathrm{n}=19)$ of the blocks were poorly oriented, which made their diagnosis difficult. Finally, it was observed that in $7.9 \%(n=9)$ of the biopsy paraffin blocks the tissue was gradually depleted as a greater number of levels were examined, which made diagnosis difficult.

Upon examination of the second cut on the second level of the biopsy paraffin blocks, we observed that $37.5 \%$ (57 paraffin blocks) of the cases differed from the initial diagnoses. It was observed that $46.9 \%$ (15 paraffin blocks) of the cases with an initial HPVI diagnosis corresponded to CIN I on the second evaluation. For the cases with an initial diagnosis of CIN I, 12.9\% (4 paraffin blocks) were changed to CIN II on the second evaluation. For the cases with an initial diagnosis of CIN II, 26.7\% (8 paraffin blocks) changed to CIN III, and 1 case (3.3\%) was changed to in situ CC (Figure 3). In the case of the samples with an initial CIN III diagnosis, 31\% (9 paraffin blocks) of the samples changed to in situ CC. Regarding the cases with an initial diagnosis of in situ CC, 7.1\% (1 paraffin block) changed to a diagnosis of invasive CC. Finally, the cases initially diagnosed as invasive cancer did not change $(\mathrm{p} \leq 0.05)$ (Figure 3$)$.

By examining the third cut on the third level of the biopsy paraffin blocks, we observed that there were no

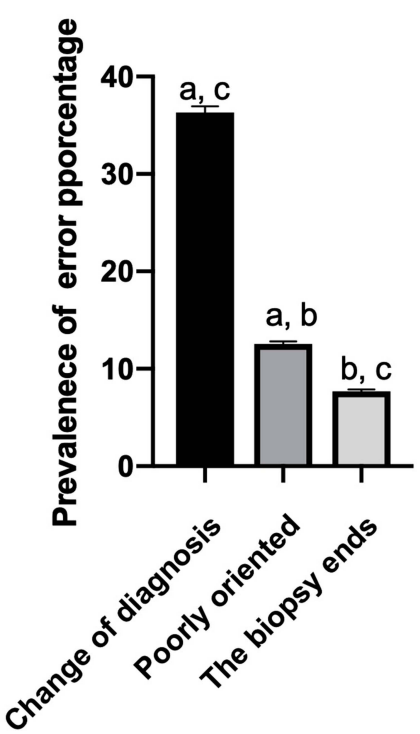

Figure 2 Main changes and results based on the examination of the different levels of the paraffin blocks. a is the group change of diagnosis, $36.8 \%$; $b$ is the poorly oriented $12.5 \%$, and $\mathrm{c}$ is the biopsy ends, $7.6 \%$. The groups a, b and $\mathrm{c}$ had statistical differences $p \leq 0.05$

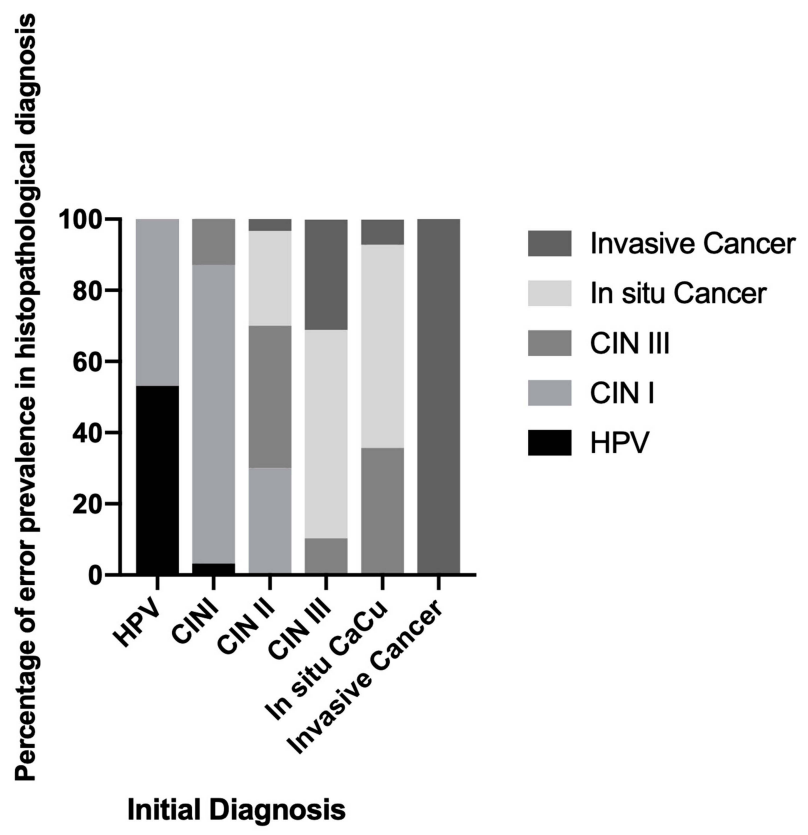

Figure 3 Change from the initial diagnosis based on evaluation of the second level of the paraffin block (value of $p \leq 0.05$ ). $n=120$.

additional diagnosis changes beyond those observed in the second cut in $34.2 \%$ of the samples (52 paraffin blocks). The initial diagnosis of CIN I was changed to HPVI in $6.5 \%$ of the samples ( 2 paraffin blocks), decreasing the percentage of CIN I to $80.6 \%$ (25 paraffin blocks) (Figure 4). For cases with an initial diagnosis of CIN II, it was now found that $20 \%$ (6 paraffin blocks) corresponded 
to a CIN I diagnosis. For the cases with an initial diagnosis of CIN III, $6.9 \%$ (2 paraffin blocks) were changed to a CIN II diagnosis. In the cases with an initial diagnosis of $\mathrm{CC}$ in situ, $7.1 \%$ (1 paraffin block) were changed to invasive CC. Finally, the original invasive CC cases did not change after evaluation of the third level (Figure 4) $(\mathrm{p} \leq 0.05)$.

The analysis of the slides of the fourth cut in the fourth level of the biopsy paraffin blocks showed that $32.9 \%$ (50 paraffin blocks) of the cases differed from the initial diagnoses. In the cases originally diagnosed as CIN II, $16.7 \%$ (5 paraffin blocks) changed to CIN I. It is noteworthy that the diagnoses did not change completely from the third level to the fourth level (Figure 5; all of the aforementioned with $\mathrm{p} \leq 0.05$ ). To observe the concordance of the initial diagnosis (pathologist of the institution) and the final diagnosis (pathologist that acted as the gold standard), a kappa coefficient was calculated; the kappa value of 0.673 indicated that there is a correlation between the two readings of the lamellae with $\mathrm{p} \leq 0.05$; that is, the two pathologists were making a correct reading, and the difference was in the level of the cut (Table 1).

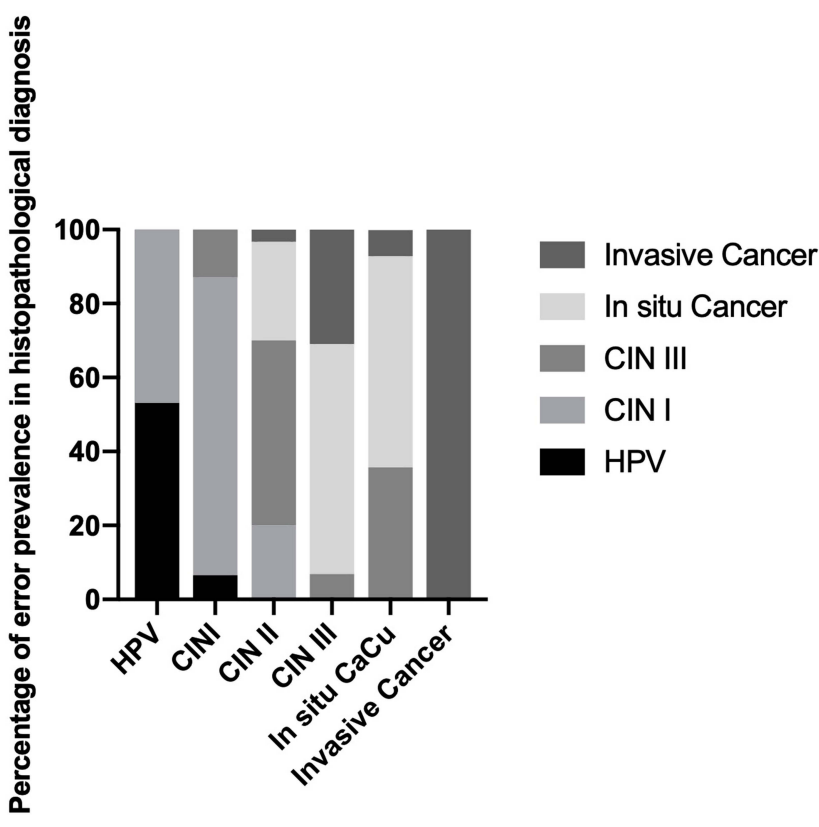

Initial Diagnosis

Figure 4 Change from the initial diagnosis based on evaluation of the third level of the paraffin block (value of $p \leq 0.05$ ). $n=120$.

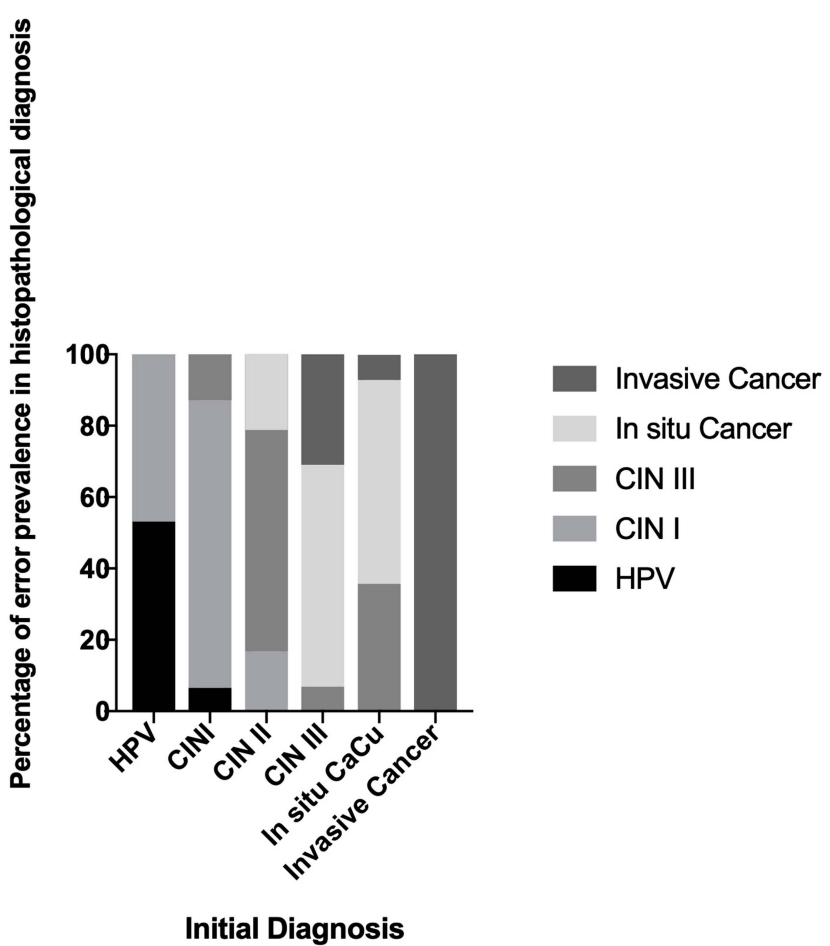

Figure 5 Change from the initial diagnosis based on evaluation of the fourth level of the paraffin block (value of $p \leq 0.05$ ). $n=120$.

\section{Discussion}

Laboratory confirmation of any positive screening test result is critical for quality assurance and optimal patient care. The correlation of cervicovaginal PAP tests diagnosed with dysplasia, with their subsequent cervical biopsies, is an important part of quality assurance in the cytology laboratory. There are several reasons that support the observations aforementioned; one of them is that dysplasia may be sampled at the time of colposcopy, but the dysplasia may not be apparent on the resulting slide because of inadequate sectioning of the paraffin block. ${ }^{2}$

It was observed that $12.5 \%$ of the samples were not included in the paraffin block in a well-oriented manner for analysis, since the stroma was placed towards the edge where the pathologists begin to make the cuts; as such, one sees blood vessels or glands instead of the epithelial tissue that must be analyzed when observing the cut under the microscope. The orientation must be such that the resistance that the tissue offers to the blade goes from a minor degree to a greater one so that the harder tissues do not compress the soft ones, and irregularities that alter the observation are avoided. ${ }^{2}$ In the case of samples that come from cervical biopsies, it is important to be able to see the epithelium, which is where the lesions are located, to make an adequate diagnosis. ${ }^{13-15}$ 
Table I Concordance of Histopathological Diagnoses $(p \leq 0.05) . n=120$ Direct Source

\begin{tabular}{|c|c|c|c|c|c|c|c|c|}
\hline & & \multicolumn{7}{|c|}{ Diagnosis 2} \\
\hline & & HPV & CIN I & CIN II & CIN III & Cancer in situ & Invasive Cancer & Total \\
\hline \multirow[t]{7}{*}{ Diagnosis I } & HPV I & 16 & 2 & 0 & 0 & 0 & 0 & 18 \\
\hline & CIN I & 13 & 33 & 4 & 0 & 0 & 0 & 50 \\
\hline & CIN II & 0 & 8 & 6 & 4 & I & 0 & 19 \\
\hline & CIN III & 0 & 2 & 2 & 25 & I & 0 & 30 \\
\hline & Cancer in situ & 0 & 0 & 0 & 0 & 15 & 3 & 18 \\
\hline & Invasive Cancer & 0 & 0 & 0 & 0 & 0 & 17 & 17 \\
\hline & Total & 29 & 45 & 12 & 29 & 17 & 20 & 152 \\
\hline
\end{tabular}

In our study, it was observed that there were changes in more than a third of the 152 paraffin blocks analyzed in relation to the initial diagnosis; this was caused by an increase in the number of cuts. In the work performed by Goldband et al, ${ }^{3}$ it was found that, of 436 consecutive cone biopsies initially reported as negative, the final diagnoses after reevaluation were positive $(\mathrm{n}=21)$, unsatisfactory $(\mathrm{n}=27)$ and true negative $(\mathrm{n}=51)$, with one case excluded because of insufficient material for review. ${ }^{3}$

In contrast, Joste et al (2005) ${ }^{1}$ evaluated initially negative biopsy slides for four histological features: chronic cervicitis, acute cervicitis, mucosal erosion, and squamous atypia. Twenty-seven biopsies (24.3\%) showed the presence of a squamous intraepithelial lesion (SIL) at deeper levels. The presence of squamous atypia was significantly associated with the presence of dysplasia deeper in the block $(\mathrm{p}=<0.002){ }^{1}$

In addition, in the present investigation, we found that by increasing the number of cuts made in the biopsy paraffin block, there were changes observed in all of the initial diagnosis types of HPV infection and all of the other injury types, with percentages that turned out to have statistical significance $(\mathrm{p}<0.05 ; 95 \% \mathrm{CI})$. Joste and colleagues found that the complete sectioning of paraffin blocks with review of intermittent levels led to a $24.3 \%$ increase in the detection of SIL in noncorrelated cervical biopsies. They claim that the number of additional cervical dysplasias revealed by additional sectioning was greater than anticipated.

In his study called "Deeper examination of negative colorectal biopsies", Luo ${ }^{15}$ studied specimens from colorectal biopsies of putative lesions for which initial sections lacked polyps. They determined the incidence of polyps within additional sections and whether the presence of lymphoid aggregates within initial sections excluded the presence of polyps within additional sections. They studied 83 specimens from colorectal biopsies, and the examination of additional sections revealed polyps for nearly $10 \%$ of specimens. Furthermore, exhaustive sectioning might have revealed more polyps. ${ }^{4}$

Our results differ from the clinical practice guidelines for gynecological pathology, which specify that at least three cut-off levels are required for diagnostic accuracy, since we show that the increase in the number of cuts changes the diagnosis in favor of higher grade injuries.

It should be mentioned that, in the present study, the professionals who analyzed the biopsies (double blind) were certified pathologists; however, staff training should be considered $^{5}$ since, in some studies, such as the study conducted by Gutíerrez-Enríquez, ${ }^{6}$ differences in cytological diagnosis between cytotechnologists and pathologists were evident. They found that, in their evaluations of epithelial abnormalities, only one of the cytotechnologists had significant concordance with the pathologists; however, the concordance was low. In the case of glandular abnormalities, only one of the cytotechnologists had no significant concordance with the pathologists. In this study, the cytotechnologist, who had a high level of academic training, performed the staining procedure, analysis and interpretation of the smears properly ${ }^{13-15}$ It was observed that experience is apparently an important factor in the analysis and interpretation of smears, as the cytotechnologist who had more than 15 years of experience performed the procedures adequately. The authors concluded that the concordance between cytotechnologists and pathologists is below the required minimum level set by national and international standards. ${ }^{6}$

\section{Conclusion}

Multilevel examination has been cited as a means to avoid diagnostic error. However, the review of the literature 
reveals very little research on the optimal number of levels necessary to carry out the diagnosis with accuracy. More research that includes comparative studies of histopathological diagnoses in suspected cervical cancer should be performed to provide a timely and effective diagnosis for the benefit of women with pre-neoplastic and neoplastic lesions; a correct early diagnosis could potentially save lives.

\section{Disclosure}

The authors report no conflicts of interest in this work.

\section{References}

1. Joste NE, Wolz M, Pai RK, Lathrop S. Noncorrelating pap tests and cervical biopsies: histological predictors of subsequent correlation. Diagn Cytopathol. 2005;32:310-314. doi:10.1002/dc.20172

2. Rolls G. Microtomía y preparación de la sección en parafina. Scientia Leica Microsystems' Education Series; 2019:11. Available from: www.leica-microsystems.com.

3. Golbang P, Scurry J, de Jong S, et al. Investigation of 100 consecutive negative cone biopsies. BJOG. 1997;104:100-104. doi:10.1111/j.14710528.1997.tb10658.x

4. Wu ML-C, Dry SM, Lassman CR. Deeper examination of negative colorectal biopsies. Am J Clin Pathol. 2002;117:424-428. doi:10.1309/ VBYP-UT2E-V198-M1TQ

5. Herrera Pérez MA, Cirión Martínez GR, Sanabria Negrín JG. Impacto de la capacitación en la mejora continua de la calidad del diagnóstico citológico. Revista de Ciencias Médicas de Pinar del Río. 2010;14:79-91.
6. Gutiérrez-Enríquez SO, Chávez-Hernández L, Terán-Figueroa Y, et al. Concordance in the interpretation of cervical cytology for the early diagnosis of cervical cancer. Open $J$ Obstet Gynecol. 2016;6:714-724. doi:10.4236/ojog.2016.612089

7. Fadare O, Rodriguez R. Squamous dysplasia of the uterine cervix: tissue sampling-related diagnostic considerations in 600 consecutive biopsies. Int J Gynecol Pathol. 2007;26(4):469-474. doi:10.1097/ pgp.0b013e318038154d

8. Heatley MK. How many histological levels should be examined from tissue blocks originating in cone biopsy and large loop excision of the transformation zone specimens of cervix? J Clin Pathol. 2001;54 (8):650-651. doi:10.1136/jep.54.8.650

9. International Agency for Research on C. GLOBOCAN 2012: estimated cancer incidence, mortality and prevalence worldwide in 2012. 2012.

10. Brown L, Andrew A, Hirschowitz L, Millan D. Tissue pathways for gynaecological pathology. London: The Royal College of Pathologists. 2008.

11. Arbyn M, Anttila A, Jordan J, et al. European guidelines for quality assurance in cervical cancer screening-summary document. Ann Oncol. 2010;21(3):448-458. doi:10.1093/annonc/mdp471

12. College of American P. Cancer protocol templates. CAP Web site. 2014 [cited March 31, 2015]. Available from: http://www.cap.org/web/home/ resources/cancer-reporting-tools/cancer-protocol-templates.

13. Slaoui M, Fiette L. Histopathology Procedures: From Tissue Sampling to Histopathological Evaluation. In: Gautier JC, editor. Drug Safety Evaluation, Methods and Protocols. Totowa, NJ: Humana Press; 2011;691:69-82.

14. Vega MSMQ, Gómez JFC, Bastidas M, Márquez L, Pons JP. Detección y tipificación de virus del papiloma humano (VPH) mediante PCR-RFLP. Obstetricia y Ginecología. 2008;68(1):25-31.

15. Luo YV, Prihoda TJ, Sharkey FE. Number of levels needed for diagnosis of cervical biopsies. Arch Pathol Lab Med. 2002;126 (10):1205-1208

\section{Publish your work in this journal}

Cancer Management and Research is an international, peer-reviewed open access journal focusing on cancer research and the optimal use of preventative and integrated treatment interventions to achieve improved outcomes, enhanced survival and quality of life for the cancer patient.
The manuscript management system is completely online and includes a very quick and fair peer-review system, which is all easy to use. Visit http://www.dovepress.com/testimonials.php to read real quotes from published authors. 